\title{
Characterization of III/V Semiconductors on Silicon by Analyzing 4D-STEM Data with Convolutional Neural Networks
}

Damien Heimes ${ }^{1}$, Jonas Scheunert ${ }^{2}$, Andreas Beyer ${ }^{1}$, Jürgen Belz ${ }^{2}$, Saleh Firoozabadi ${ }^{3}$ and Kerstin Volz ${ }^{1}$

${ }^{1}$ Materials Science Centre and Department of Physics, Philipps University Marburg, Hans-MeerweinStraße 6, Marburg, 35032, Germany, Germany, ${ }^{2}$ STRL, University of Marburg, Germany, ${ }^{3}$ Materials Science Centre and Department of Physics, Philipps University Marburg, Hans-Meerwein-Straße 6, Marburg, 35032, Germany, Marburg, Hessen, Germany

$\mathrm{Si}$ based devices dominate our everyday life. Limitations of pure $\mathrm{Si}$, like its indirect band gap, could be overcome by the integration of III/V semiconductors. Growing III/V compounds on Si is challenging, e.g. due to the formation of charges at interfaces and antiphase defects [1]. Hence characterization on the nanoscale is crucial.

Apart from well established methods like high-angle annular dark-field (HAADF) scanning transmission electron microscopy (STEM), which have been applied for the characterization of III/V Semiconductors on $\mathrm{Si}$ [1], there are new possibilities available: In recent years the upcoming of fast pixelated detectors in STEM made it possible to generate datasets containing the convergent beam electron diffraction (CBED) pattern at every probe position [2,3]. This method is referred to as 4D-STEM.

Compared to data from annular detectors, which consist of only one intensity value per scan point, 4DSTEM entails a huge increase in the data amount and it already is a versatile method which has many applications. These include, e.g., virtual annular detectors [4], ptychography [4], and the measurement of electric fields $[2,3]$. However, handling this big data is challenging.

A new upcoming method, which has proven in recent years to be able to extract valuable information from 4D-STEM datasets, are convolutional neural networks (CNN). Once they are trained properly, they facilitate the determination of many parameters, e.g., thickness [5], tilt angle [5], and crystal structure [6].

The objective of this study is to train CNNs which are able to evaluate 4D-STEM data of III/V compounds on Si. It shall especially facilitate the evaluation of atomic structures at interfaces between substrate and III/V compounds. This means the requirements to the capabilities of the CNNs are to include the correct evaluation for substrate and III/V compound as pure, individual materials on the one hand, and to also extract correct information at interfaces for both materials together.

The compound Gallium Phosphide (GaP) grown on Si serves as a first case study. For this material system CBED training datasets are generated via STEM multislice simulations [7, 8]. The datasets are augmented in multiple ways, including several random affine transformations and experimental noise.

Single CNNs are trained, each to extract a certain quantity from the 4D data, including thickness, tilt angle, rotation, finding the $\mathrm{CBED}$ center, and segmentation (element determination).

Figure 1 shows results of a CNN which was trained to segment data pixelwise into atomic columns of the right elements and a background. The left-hand side depicts HAADF images and the right-hand side the corresponding segmentation. Predictions for the pure materials Si and GaP for experimental images are shown, as well as predictions for a simulated image of a $\mathrm{Si} / \mathrm{GaP}$ interface. In all cases it is segmented correctly between atomic columns and background. The right elements also are predicted both for pure materials as well as for the interface. This already proves that training CNNs for multiple materials is feasible. 
The second example presented here is a $\mathrm{CNN}$ which was trained to determine specimen thickness from position averaged CBED (PACBED) images for both $\mathrm{Si}$ and GaP. Figure 2 shows 3 PACBEDs of different specimen thickness and the thickness predictions from the CNN. The differences in the angular range in the images stem from augmenting the data with random crops. For simulated test data, the thickness was always predicted correctly with a maximum error of $2.5 \mathrm{~nm}$ and the achieved validation accuracy is 99.2 $\%$.

Applying CNNs to 4D-STEM data is a promising method, which has the capability to improve the characterization of III/V semiconductors on $\mathrm{Si}$, also for the special challenges concerning interfaces.

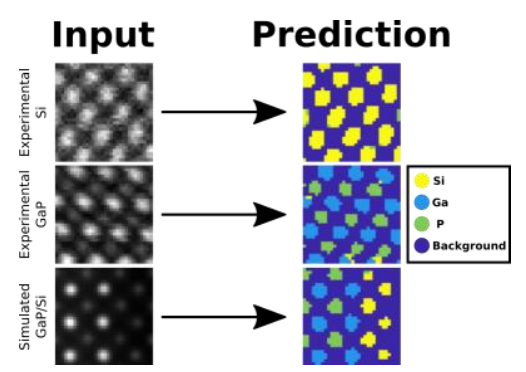

Figure 1. Figure 1: Input for CNN, which was trained for segmenting, together with the corresponding predictions.
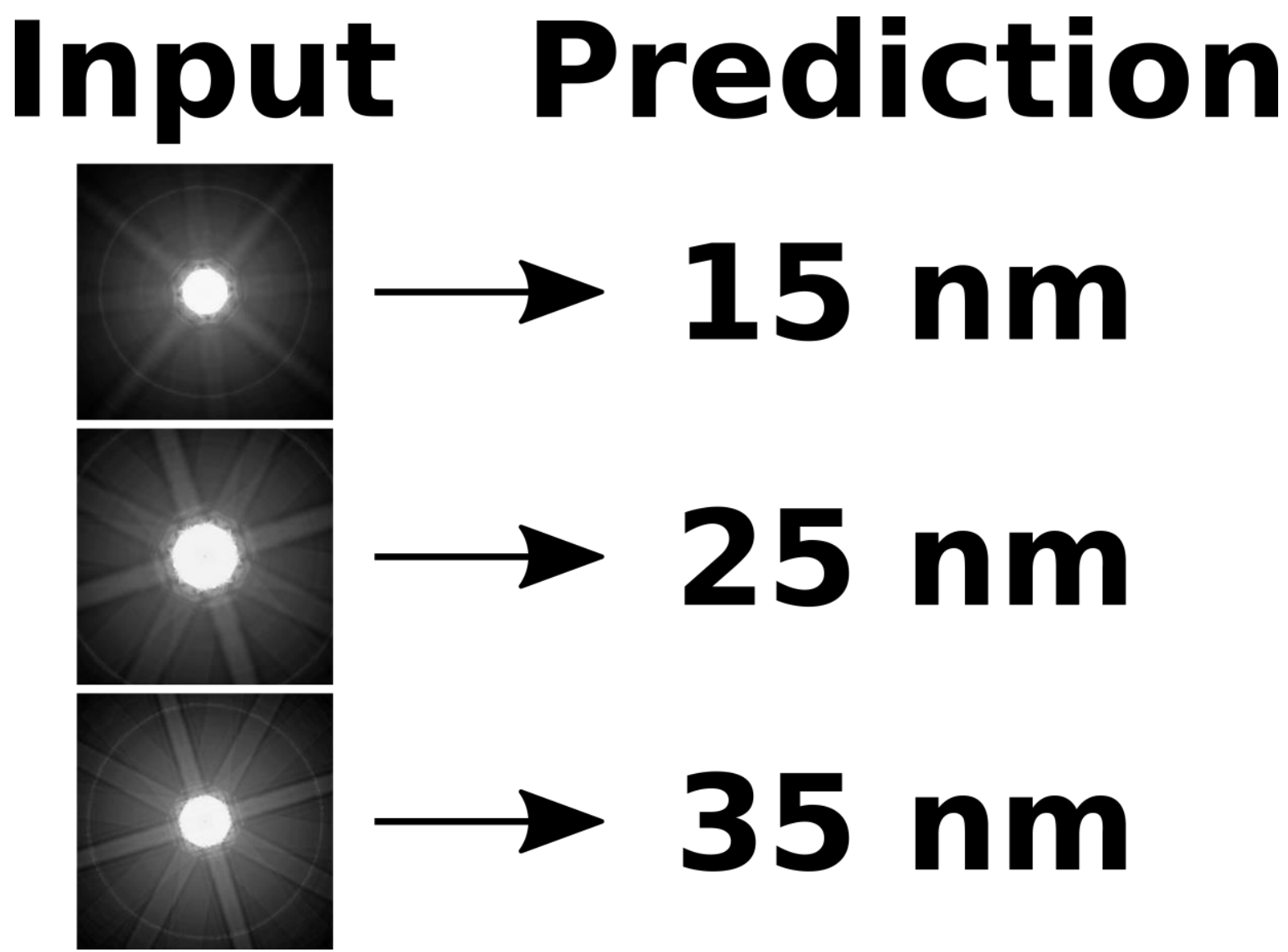

Figure 2. Figure 2: Input for CNN, which was trained to predict specimen thickness from PACBED patterns, and the corresponding predictions. Angular ranges differ, because augmentations include a random centered crop. 


\section{References}

[1] Beyer, A., \& Volz, K. (2019). Advanced Electron Microscopy for III/V on Silicon Integration. Advanced Materials Interfaces, 6(12), 1801951.

[2] Beyer, A., Munde, M. S., Firoozabadi, S., Heimes, D., Grieb, T., Rosenauer, A., Müller-Caspary, K., \& Volz, K. Quantitative Characterization of Nanometer-Scale Electric Fields via Momentum-Resolved STEM. Nano Letters (accepted 2021-02-19).

[3] Gao, W., Addiego, C., Wang, H., Yan, X., Hou, Y., Ji, D., Heikes, C., Zhang, Y., Li, L., Huyan, H., Blum, T., Aoki, T., Nie, Y., Schlom, D. G., Wu, R., \& Pan, X. (2019). Real-space charge-density imaging with sub-ångström resolution by four-dimensional electron microscopy. Nature, 575(7783), 480-484.

[4] Ophus, C. (2019). Four-Dimensional Scanning Transmission Electron Microscopy (4D-STEM): From Scanning Nanodiffraction to Ptychography and Beyond. Microscopy and Microanalysis, 2019, 563-582. [5] Xu, W., \& LeBeau, J. M. (2018). A deep convolutional neural network to analyze position averaged convergent beam electron diffraction patterns. Ultramicroscopy, 188, 59-69.

[6] Aguiar, J. A., Gong, M. L., Unocic, R. R., Tasdizen, T., \& Miller, B. D. (2019). Decoding crystallography from high-resolution electron imaging and diffraction datasets with deep learning. Science Advances, 5(10), 1-10.

[7] Oelerich, J. O., Duschek, L., Belz, J., Beyer, A., Baranovskii, S. D., \& Volz, K. (2017). STEMsalabim: A high-performance computing cluster friendly code for scanning transmission electron microscopy image simulations of thin specimens. Ultramicroscopy, 177, 91-96.

[8] Kirkland, E. J. (2020). Advanced Computing in Electron Microscopy: Third Edition. Springer Nature Switzerland AG. 